\title{
Preface
}

\section{Materials for Energy Applications}

With this special issue of Metallurgical and Materials Transactions A, we begin what we plan as a series of issues that focus on Materials for Energy Applications. We feel that articles such as the ones in this issue fit well within our scope. Structure/property studies on specific materials that are used in energy conversion or storage applications are the studies that we seek to publish in these issues. Since we also cover materials processing, innovative ways of producing metals and other materials that can be done with less energy or less pollutants are also of interest to us. The green cover is a simple way of reminding the readers that the issue in their hands is an energy-related issue of the journal.

Dr. K. Scott Weil of Owens-Illinois Corporation has acted as Guest Editor for this issue. Please feel free to contact me (mettrans@andrew.cmu.edu) if you have any questions about our green issues or if you have a paper you wish to submit for review. We also will publish clusters of articles on specific materials applications such as we published in the April 2011 issue of MMTA (Materials for Nuclear Energy - Chairs: M.G. Burke and M.A. Burke and Materials for Fossil Energy System-Chair: Seetharaman Sridhar). If you would like to put together such a cluster, contact either Scott or me and we can discuss your request if we feel it to be appropriate.

Please feel free to let us know what you think of this green issue of MMTA.

David E. Laughlin

Principal Editor

Over the past thirty years, worldwide consumption of energy has expanded at a dramatic rate. In many industrialized countries, fossil fuels - crude oil, coal, and natural gas-represent three-quarters or more of the energy presently consumed. Keeping up with the growing global demand for fossil energy has become an increasingly complex issue fraught with innumerable factors both political and technical in nature. In particular, the environmental impact and geopolitical consequences of our heavy reliance on fossil fuels will continue to be ever more conspicuous and problematic. As a result, there has been a growing desire in many nations to take advantage of native, sustainable energy sources and utilize current sources of energy more efficiently to reduce their dependencies on foreign sources. To do this in a cost-effective and ecologically conscious manner will require the emergence of new energy production, storage, and conservation technologies. Critical to many of these will be materials innovations that enable higher power generating performance, greater energy conversion and transmission efficiencies, extended operating life and more demanding service conditions, higher capacity energy storage, and reduced emissions.

Metallurgical and Materials Transactions $A$ is developing special issues of the journal that will highlight the latest research on these topics and others being conducted at the intersection of the materials and energy fields. This current issue contains papers that discuss the latest findings on: new materials development for energy generation, storage, or conservation; advanced fabrication and synthesis processes for materials used in energy devices and power generation equipment; lower energy consumption approaches to materials production; materials degradation and environmental interaction issues; and materials engineering performance in prototypic power generation systems.

\section{K. Scott Weil}

Guest Editor 